\title{
Genetic differentiation at species level in the Neotropical army ant Labidus praedator
}

\author{
M. B. Barth ${ }^{1,2}$, R. F. A. Moritz ${ }^{1,3}$ and F. B. Kraus ${ }^{4}$ \\ ${ }^{1}$ Department of Biology, Martin-Luther-University Halle-Wittenberg, Hoher Weg 4, 06099 Halle (Saale), \\ Germany \\ 2 Present Address: DNA-Laboratory, Museum of Zoology, Senckenberg Natural History Collections Dresden, \\ Königsbrücker Landstr. 159, 01109 Dresden, Germany \\ ${ }^{3}$ Department of Zoology and Entomology, University of Pretoria, Pretoria 0002, South Africa \\ ${ }^{4}$ Department of Laboratory Medicine, University Hospital Halle, Ernst Grube Str. 40, 06120 Halle (Saale), \\ Germany \\ *Correspondence to benjamin.barth@zoologie.uni-halle.de
}

\begin{abstract}
The nomadic, swarm-raiding army ant Labidus praedator (Smith, 1858) is an important arthropod predator in the Neotropics with a strong ecological impact on invertebrate communities. However, despite its high abundance and ubiquity over a large distribution range, it received relatively little scientific attention. Moreover, the taxonomic status is confusing because some morphological descriptions point towards the co-occurrence of several distinct taxa which are lumped together as $L$. praedator in most classical keys. Yet, clarifying genetic studies are lacking. Here, we show strong genetic differentiation within an L. praedator population in Mexico. Both microsatellite genotype patterns and phylogenetic analyses (concatenated nuclear and mtDNA sequences, including the coxl genetic barcoding region), reveal the occurrence of two strongly isolated lineages. Colonies from the very same location, clearly identified as the same species (L. praedator) according to classical morphological keys, exhibit an extremely high average sequence divergence (9.7-12.8 \%), which was well in the range of divergence among GenBank sequences from other Labidus species. Thus, our data very likely show genetic differentiation at species level or cryptic speciation within L. praedator, which should be recognized when investigating biodiversity and ecological importance of army ants (or other arthropods) in the Neotropics.
\end{abstract}

\section{Keywords}

Cryptic speciation, Genetic barcoding, Genetic diversity, Maximum likelihood, Microsatellites, Sequence divergence

\section{Introduction}

The nomadic, swarm-raiding army ants are important predators with a strong ecological impact in the Neotropics since they efficiently harvest a broad spectrum of invertebrates (Franks 1982; Franks and Bossert 1983; Gotwald 1995; Kronauer 2009). One of the most abundant and ubiquitous swarm-raiders is Labidus praedator (Smith, 1858), with extremely large colonies (probably over 1,000,000 individuals) and a wide distribution range spanning from Mexico to northern Argentina (Rettenmeyer 1963; Schneirla 1971; Watkins 1976; Longino 2005). Raids of L. praedator are frequent in the Neotropics (Kaspari and O'Donnell 
2003; O'Donnell et al. 2007) and can deplete invertebrate biomass in the litter by up to $75 \%$ (Kaspari et al. 2011). Despite this particularly strong ecological impact, most research on army ant biology has been based on swarm-raiding species of the genus Eciton, while $L$. praedator received much less attention. One reason might be its cryptic life history with unsteady and partially underground foraging activity (Rettenmeyer 1963; Schneirla 1971; Fowler 1979). Another problem is an unclear taxonomic status of L. praedator subspecies and local variants. For example, Borgmeier (1955) distinguishes two partially sympatric subspecies (L. p. s. str. and L. p. sedulus), but with weak morphological support for the worker caste (Rettenmeyer 1963; Longino 2005), and Longino (2005) suggests a morphologically distinct local variant (L. sp. JTL-001) from Costa Rico. Such ambiguities may in fact indicate cryptic speciation, which seems to be common in ants and needs to be addressed in biodiversity studies (Smith et al. 2005; Seifert 2009; Fournier et al. 2012). Given the ecological significance of $L$. praedator, it appears to be most timely to assess the intraspecific genetic divergence of these army ants using DNA tools. We do this in a case study for a Mexican L. praedator population, analyzing the genetic structure by microsatellite marker genotyping and sequencing fragments of the nuclear and mitochondrial genome, including parts of the genetic barcoding region of the cytochrome $c$ oxidase subunit I (coxl) (Hebert et al. 2003a, b).

\section{Materials and methods}

Samples of 79 to 128 workers were taken from four L. praedator colonies between 2005 and 2008 in Chiapas, Mexico within a range of $10 \mathrm{~km}$ around the city of Tapachula (Table 1), preserved in $95 \%$ ethanol and stored at $-20^{\circ} \mathrm{C}$. Queens of army ants are highly polyandrous and mate with about 20 males from the surrounding colonies (Kronauer et al. 2007; Jaffé et al. 2009; Barth et al. 2014). By inferring the genotypes of the queen and the siring males from a large sample of workers of a colony, it is, therefore, possible to obtain information not only on the genotypic composition of the colony but also on the overall population structure. We used Chelex DNA extraction (Walsh et al. 1991) and standard polymerase chain reactions (PCR) to genotype all sampled workers (after removal of gasters) at nine microsatellite loci, four of which (Eb04, Eb25, Eb42, DmoD) were developed for other army ants (Denny et al. 2004; Kronauer et al. 2004), while five (Lp2, Lp4, Lp14a, Lp30, Lp38) were recently developed for L. praedator (Barth et al. 2014). Queen and male genotypes were reconstructed by Mendelian inferences and double checked with the software MATESOFT (Moilanen et al. 2004). By duplicating the queen genotypes and taking the males as homozygote diploids in the program FSTAT 2.9.3 (Goudet 1995), we achieved a diploid input file and estimated $F_{\mathrm{ST}}$ values for population differentiation (treating colonies as subpopulations). 
Table 1: Sampling data, sample size of workers $\left(n_{\mathrm{w}}\right)$, reconstructed number of males $\left(n_{\mathrm{m}}\right)$, private allelic richness $\left(A_{\text {priv }}\right)$ and GenBank accession numbers for the sequenced fragments of four L. praedator colonies in Chiapas, Mexico

\begin{tabular}{|c|c|c|c|c|c|c|c|c|}
\hline Colony & \begin{tabular}{|c|}
$\begin{array}{c}\text { Sampling } \\
\text { date }\end{array}$ \\
\end{tabular} & Sampling location & GPS & $n_{w}$ & $n_{m}$ & $A_{\text {priv }}$ & \begin{tabular}{|c|} 
GenBank \\
(coxl)
\end{tabular} & \begin{tabular}{|c|} 
GenBank \\
(28S) \\
\end{tabular} \\
\hline Cac1 & || $11 / 2005$ & $\begin{array}{l}\text { Cacahoatán, coffee } \\
\text { plantation }\end{array}$ & $\begin{array}{l}15^{\circ} 0^{\prime} 19^{\prime \prime} \mathrm{N}, \\
92^{\circ} 10^{\prime} 17^{\prime \prime} \mathrm{W}\end{array}$ & 128 & 21 & 3.85 & KP455502 & KP455506 \\
\hline Cac2 & || $11 / 2005$ & $\begin{array}{l}\text { Cacahoatán, coffee } \\
\text { plantation }\end{array}$ & $\begin{array}{l}15^{\circ} 0^{\prime} 19^{\prime \prime} \mathrm{N}, \\
92^{\circ} 10^{\prime} 17^{\prime \prime} \mathrm{W}\end{array}$ & 106 & 26 & 1.34 & KP455503 & KP455507 \\
\hline Tap & $12 / 2008$ & $\begin{array}{l}\text { Tapachula, } \\
\text { secondary forest }\end{array}$ & $\begin{array}{l}14^{\circ} 55^{\prime} 59^{\prime \prime} \mathrm{N}, \\
92^{\circ} 17^{\prime} 3^{\prime \prime} \mathrm{W}\end{array}$ & 109 & 18 & 0.80 & |KP455504 & KP455508 \\
\hline TC & $12 / 2008$ & $\begin{array}{l}\text { Tuxtla Chico, } \\
\text { agricultural } \\
\text { research centre }\end{array}$ & \begin{tabular}{|l}
$14^{\circ} 58^{\prime} 25^{\prime \prime} \mathrm{N}$ \\
$92^{\circ} 9^{\prime} 34^{\prime \prime} \mathrm{W}$
\end{tabular} & 79 & 26 & $\| 1.01$ & KP455505 & KP455509 \\
\hline
\end{tabular}

After standard phenol-chloroform DNA extraction of one additional L. praedator worker per colony, two mitochondrial (mtDNA) coxl fragments and one nuclear $28 \mathrm{~S}$ fragment were amplified using the primer pairs $\mathrm{Cl} 13 / \mathrm{Cl} 14$ and Jerry/Ben3R, and Bel28S/revBel28S, respectively, in PCR conditions as given in Brady (2003). Products were purified using the AMPure XP kit (Beckman Coulter) and sequenced (MWG-Biotech) at the forward and reverse strands. The consensus sequences were compared in a phylogenetic analysis with the homologous GenBank sequences (Brady 2003) of the Neotropical army ant species $L$. coecus, L. spininodis, Eciton burchellii, E. mexicanum, E. vagans, Nomamyrmex esenbeckii and the African Dorylus nigricans as outgroup.

All 11 sequences were aligned using the MUSCLE algorithm (default settings) as implemented in the sequence analysis software MEGA5 (Tamura et al. 2011) resulting in a total length of $1387 \mathrm{bp}$ of concatenated coxl (886 bp) and $28 \mathrm{~S}$ (501 bp) fragments (GenBank accession numbers KP455502-KP455509, Table 1). MEGA5 was then also used to perform phylogenetic estimations (complete deletion of gaps and missing data). No gaps, stopcodons or discrepancies between forward and reverse strands were contained in the coxl alignment, so that the amplification of nuclear pseudogenes (Bensasson et al. 2001) could be excluded. Based on the lowest Bayesian Information Criterion score, a General Time Reversible (GTR) model + G (Gamma shape parameter $=0.23$ ) was selected as best-fitting nucleotide substitution model for the data to construct a Maximum Likelihood (ML) phylogenetic tree using the nearest neighbor interchange algorithm (Nei and Kumar 2000; Tamura et al. 2011; Hall 2013). Likewise, a Neighbor Joining (NJ) tree was constructed, following the Tamura-Nei model estimated by the Maximum Composition Likelihood method (Tamura et al. 2004). As mtDNA and nuclear DNA sometimes represent different evolutionary signals (e.g., Rabeling et al. 2014), an ML tree was also estimated separately for the $28 \mathrm{~S}$ alignment using a Tamura-3-parameter substitution model $+\mathrm{G}=0.20$. At last, pairwise nucleotide sequence divergence was estimated following the Kimura-2-parameter (K2P) model. 
Additional sequencing of the coxl genetic barcoding region with the primers LepF/LepR (Hebert et al. 2004; Smith et al. 2014) yielded multiple peaks in the sequence chromatograms of colonies Cac2, Tap and TC indicating nuclear pseudogenes (Bensasson et al. 2001) of this fragment. We, therefore, used our $\mathrm{Cl} 13 / \mathrm{Cl} 14$ fragments, which partially overlap with the barcoding region, to achieve a final alignment of $490 \mathrm{bp}$ with eight barcoding fragments from GenBank (Smith et al. 2014) of L. praedator from Costa Rica, including variant JTL-001 and five more variants for which no morphological description is available yet. Phylogenetic analyses were performed as described above (GTR $+\mathrm{G}=0.32$, partial deletion with $90 \%$ site coverage cutoff), using L. spininodis as outgroup (13 sequences in total). All alignments and trees were deposited in the internet data bank TreeBase (http://purl.org/phylo/treebase/phylows/study/TB2:S16955).

\section{Results}

All four colonies were clearly identified as $L$. praedator for the absence of an anteroventral petiole tooth (Fig. 1), which is the character to discriminate L. praedator from other Labidus species following the classical morphological keys (Borgmeier 1955; Watkins 1976, 1982). However, those keys provide no worker characteristics to distinguish L. p. s. str. and L. $p$. sedulus. Moreover, only the online key of Longino (2005) separates the taxon L. sp. JTL-001, according to a 'smooth and shiny face' character, which may apply to our colonies Cac2, Tap and TC (Fig. 1).

In all colonies, a single queen genotype and 18-26 (22.75 \pm 1.32 SD) male genotypes could be inferred from the workers (Table 1) and used to estimate population differentiation. Surprisingly, two of nine microsatellite loci were monomorphic in colony Cac1, but had more than three alleles in the other colonies, and the seven polymorphic loci differed considerably in allele size range, resulting in a high rate of private alleles for Cac1. While the allelic richness (6.39-7.53) did not differ between colonies (one-way ANOVA; $d f=24$, $F=0.42, p=0.74)$, private allelic richness (Kalinowski 2005$)$ was highest $\left(A_{\text {priv }}=3.85\right.$ ) in Cac1 $(F=5.24, p=0.006$; Table 1$)$. Using all seven polymorphic loci resulted in considerable population differentiation of $F_{\mathrm{ST}}=0.102$ (95 \% confidence from 15,000 bootstraps, $\mathrm{Cl}$ $0.075 / 0.145)$, which dropped to $F_{\mathrm{ST}}=0.069(\mathrm{Cl} 0.035 / 0.118)$ when removing Cac1 from the analysis. Pairwise tests for Cac1 had twice as high $F_{\mathrm{ST}}$ values than between the other colonies (Table 2).

Table 2: Pairwise population differentiation $\left(F_{\mathrm{ST}}\right)$ values (above diagonal), and K2P values for sequence divergence (below diagonal) between four L. praedator colonies in Chiapas, Mexico

\begin{tabular}{|l|l||c|c|c|}
\hline & Cac1 & Cac2 & Tap & TC \\
\hline Cac1 & & 0.173 & 0.128 & 0.153 \\
\hline Cac2 & 0.097 & & 0.067 & 0.066 \\
\hline Tap & 0.097 & 0.002 & & 0.076 \\
\hline TC & 0.098 & 0.001 & 0.002 & \\
\hline
\end{tabular}

Both $F_{\mathrm{ST}}$ and $\mathrm{K} 2 \mathrm{P}$ pairwise values are far higher between colonies Cac1 and Cac2, Tap or TC than between the latter three (more than 40-fold for K2P values) 


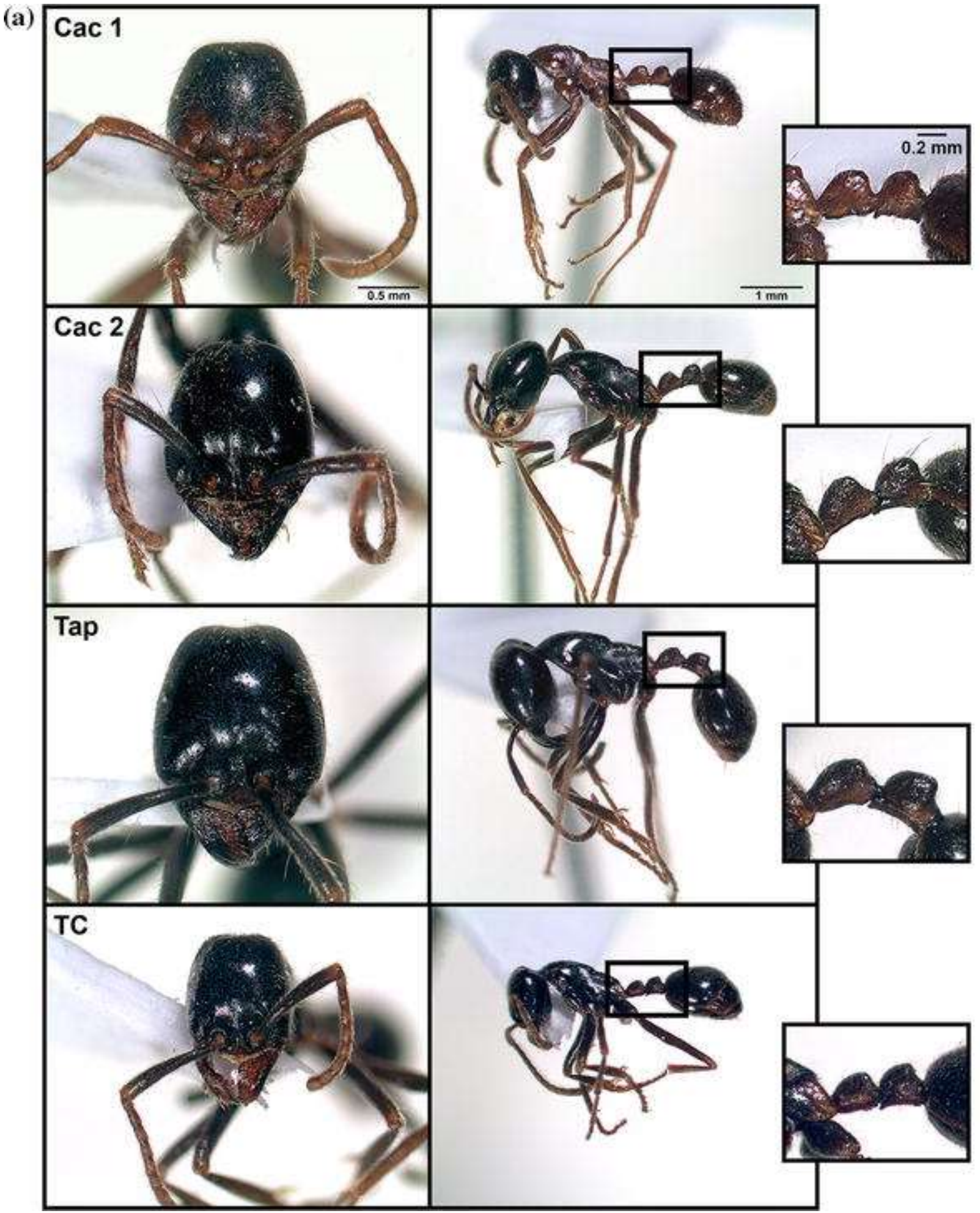


(b)
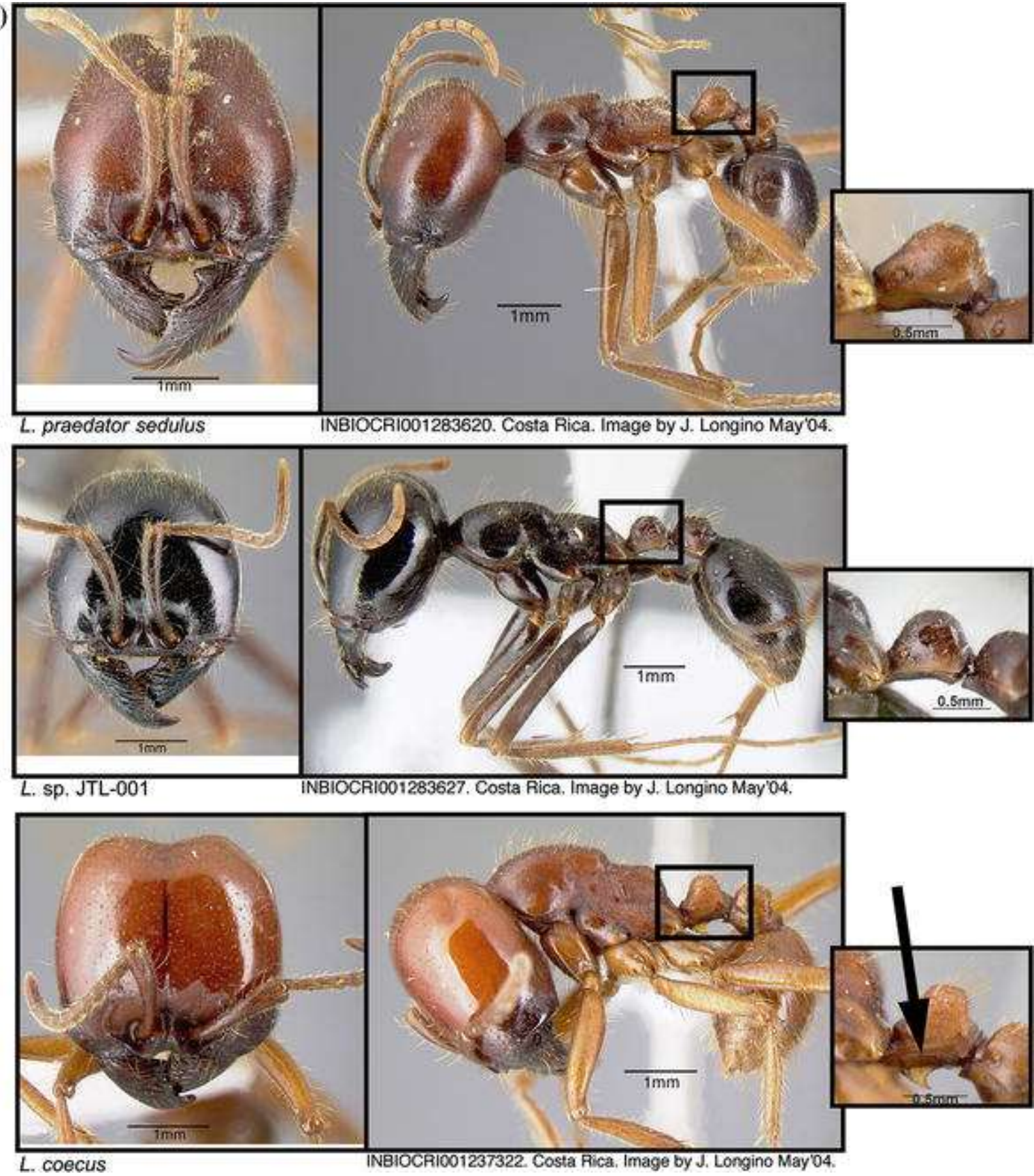

Fig. 1: Front (left images) and lateral (right images) view, and petiole (small right images) of a specimens of our four $L$. praedator colonies and $\mathbf{b}$ for comparison, images of Costa Rican specimens of $L$. p. sedulus, $L$. sp. JTL-001 and L. coecus, retrieved from Longino (2005, photo courtesy: J. Longino, with permission). L. coecus (lowest images) is the only Labidus species which co-occurs with L. praedator in Chiapas, Mexico, and can be distinguished from it by exhibiting an anteroventral petiole tooth (arrow), according to the classical morphological keys (Borgmeier 1955; Watkins 1976, 1982). Following these keys, all our specimens are clearly identified as L. praedator. However, following the 'shiny face' character in the online key of Longino (2005), the specimens of colonies Cac2, Tap and TC key out as L. sp. JTL-001

The whole 1387 bp alignment had 438 variable sites, of which 255 were parsimony informative, while the partial barcoding alignment had 151 variable sites (parsimony informative: 118), and the 28S alignment had 112 (parsimony informative: 29). For the whole and the barcoding alignment, $M L$ trees with the highest log likelihoods ( $\ln L=-4840.2$ and $\ln L=-1978.8)$, based on GTR $+G$ models, are given in Figs. 2 and 3. Both tree topologies 


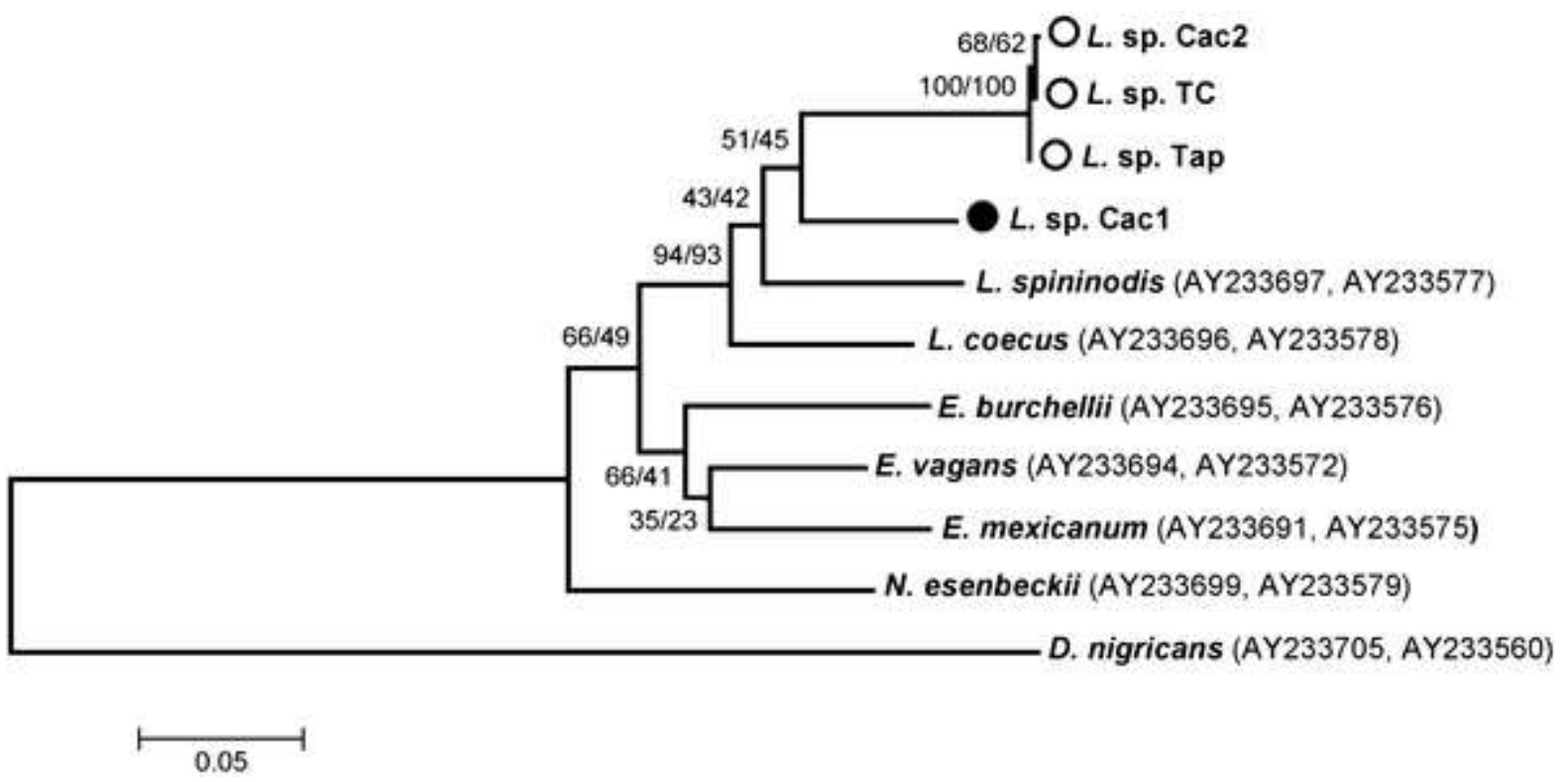

Fig. 2: Highest log likelihood ML phylogenetic tree of 11 sequences (1387 bp) of Mexican L. praedator and seven other army ant species (rooted with African D. nigricans as outgroup), based on a GTR + G model. Bootstrap values (500 replications) for the ML and NJ method are shown at the tree nodes. Branch lengths are drawn to scale (small bar) and measured in substitutions per site. Behind the taxa, colony labels are given for our samples and GenBank accession numbers (coxl, 28S) for the other species (Brady 2003). Colonies Cac2, Tap and TC (open circles) show an average sequence divergence of $0.2 \%$, but are separated from colony Cac1 (black dot) by $9.7 \%$ divergence
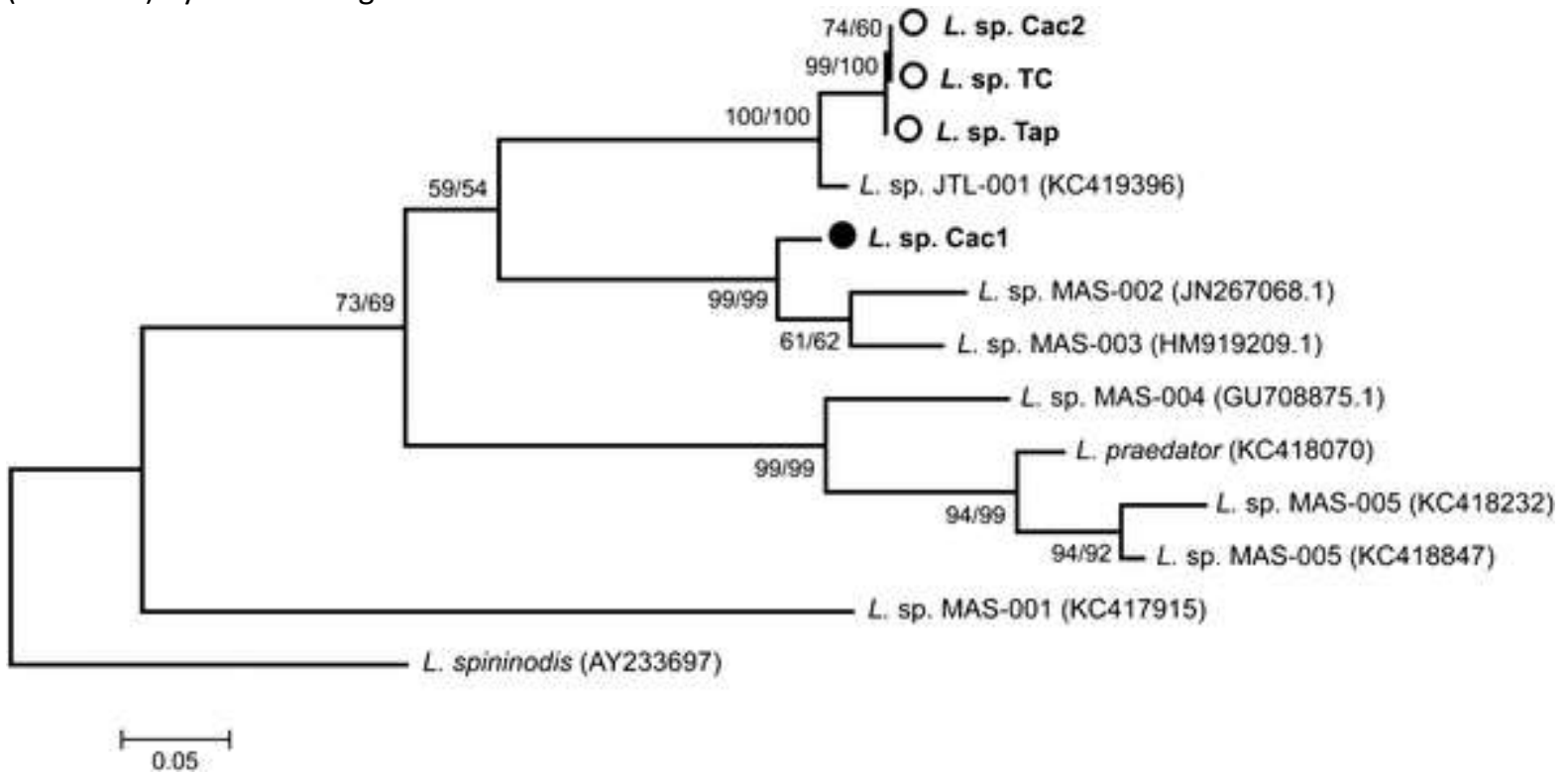

Fig. 3: Highest log likelihood ML phylogenetic tree of 13 coxl sequences (490 bp) of Mexican (bold types) and Costa Rican L. praedator lineages (rooted with L. spininodis), based on a GTR + G model. Bootstrap values (500 replications) for the $\mathrm{ML}$ and $\mathrm{NJ}$ method are shown at the tree nodes, and branch lengths in substitutions per site are drawn to scale (small bar). Colony labels or GenBank accession numbers (Smith et al. 2014) are given behind the taxa. The Mexican colonies Cac2, Tap and TC (open circles) and Cac1 (black dot) cluster in different clades with Costa Rican lineages, and show an average sequence divergence of $12.8 \%$, which is within the range (14.2\%) of overall divergence

were also obtained with NJ analyses (see TreeBase) with bootstrap values (500 replications) given at branches. The ML tree of $28 \mathrm{~S}$ supports the topology of the concatenated sequence 
tree with slightly longer branches of the latter (see TreeBase) which is expected considering the faster evolutionary rate of mtDNA (Avise 2000).

The concatenated sequence tree (Fig. 2) shows the phylogenetic relationship of our four $L$. praedator colonies and seven other army ant species. Colonies Cac2, Tap and TC cluster in one branch with very low K2P average sequence divergence $(0.2 \%)$, but are separated from Cac1 as sister group with an average pairwise divergence of $9.7 \%$ (Table 2). Average divergence is also $9.7 \%$ among all included Labidus species, and $11.7 \%$ among all species (excluding the outgroup, D. nigricans). In the barcoding phylogeny of our sequences and those of several formally undescribed Costa Rican Labidus lineages, retrieved from Smith et al. (2014), colonies Cac2, Tap and TC cluster together as sister group of L. sp. JTL-001, while colony Cac1 is much closer related to the lineages L. sp. MAS-002 and 003 (Fig. 3). Within this Labidus group, the average pairwise divergence between colony Cac1 and the other three is $12.8 \%$ (to $0.2 \%$ among the latter), while average sequence divergence is $14.2 \%$ among all lineages (excluding the outgroup).

\section{Discussion}

Our findings clearly show strong genetic differentiation among four Mexican L. praedator colonies, which may indicate distinction at species level between colony Cac1 and the other three. The lack of polymorphism of two otherwise highly diverse microsatellite loci and the particularly high rate of private alleles among all 21 males and the queen of Cac1 is highly unlikely a result of chance alone. The non-detection error of males (probability of genotypic identity by chance, Boomsma and Ratnieks 1996) is only $3.5 \%$ for the two loci, and the nonsampling error is 0.53 on average, according to fitted Poisson distributions. Also population fragmentation can hardly explain this pattern because the well flying army ant males easily allow for gene flow within our sampling area (Berghoff et al. 2008; Jaffé et al. 2009; Barth et al. 2013), and colonies Cac1 and Cac2 were even sampled in the very same time and place (Table 1). Thus, our results suggest two sympatric, but strongly reproductively isolated $L$. praedator lineages within our samples, which are, however, hardly distinguishable with the classical morphological keys (Borgmeier 1955; Watkins 1976, 1982) (Fig. 1).

This is also confirmed by phylogenetic analyses. Even though some branches contain only weak bootstrap values (probably due to our restricted sampling), particularly the separate clustering of colonies Cac2, Tap and TC in the whole sequence tree (Fig. 2) and the clustering of those colonies and Cac1 within different clades in the coxl barcoding tree (Fig. 3) is well supported. Moreover, our whole sequence ML tree (Fig. 2) is well confirmed by the army ant phylogeny of Brady (2003). However, L. praedator was not included in this phylogeny, and shows strong intraspecific sequence divergence, especially among the 490 bp barcoding fragments of recently published lineages (Smith et al. 2014) from Costa Rica (Fig. 3). Interestingly, according to the latter data, our Mexican lineages seem to be more closely related to different Costa Rican lineages than to each other, suggesting the occurrence of several strongly isolated sympatric lineages across the very large distribution range of $L$. praedator.

Average pairwise sequence divergence of $9.7 \%$ (whole alignment) between colony Cac1 and colonies Cac2, Tap and TC is over 40 -fold higher than the average divergence of $0.2 \%$ 
among the latter (Table 2), which is clearly in the range of the more distant Labidus species from GenBank (also $9.7 \%$ ). This holds as well for the cox/ barcoding fragments, while divergence becomes even more pronounced with $12.8 \%$ on average between our Mexican lineages ( $14.2 \%$ over all lineages). Though deep coxl divergence within some ant species seems not to be unusual (Smith et al. 2005; Fisher and Smith 2008), and particularly in army ants restricted maternal dispersal may foster mtDNA differentiation (Berghoff et al. 2008; Barth et al. 2013), the inclusion of nuclear 285 fragments shows that our results are not an artifact of mtDNA divergence only. Taking the $28 \mathrm{~S}$ phylogeny alone not only confirms the whole sequence tree topology but still results in a divergence of $3.7 \%$ between colony Cac1 and the other three, compared to $0.0 \%$ among the latter (2.4\% among all Labidus species). This is also supported by the population genetic differentiation pattern of nuclear microsatellite data. Our results, therefore, easily meet the requirements of the genetic barcoding approach for species discrimination with thresholds of maximal $2 \%$ intra- and a minimal tenfold higher interspecific divergence for the coxl region (Hebert et al. 2003a, b, 2004). Thus, the observed divergence very likely reflects differentiation at species level within L. praedator.

Although the subspecies $L$. p. sedulus, described on the basis of a male specimen from Colombia, has not been recorded for Mexico (Borgmeier 1955), we cannot exclude that we detected this type here because no prior genetic data exist. Morphologically, this subspecies can only be identified by the males or the largest soldier workers which we had not sampled, and which are difficult to obtain (Rettenmeyer 1963, p. 403). The variant L. sp. JTL001, since it was first mentioned for Costa Rica, has been widely recorded throughout Central America including Mexico (Longino 2005; www.antweb.org). According to the 'shiny face' character, the three Mexican colonies Cac2, Tap and TC may belong to this or a closely related taxon (Fig. 1), which is also suggested by the coxI ML tree (Fig. 3). For the MAS variants from Smith et al. (2014), no morphological descriptions exist so far. However, the present study did not intend to cover the entire spectrum of morphological and genetic variance in L. praedator. Instead, our data show deep phylogenetic divergence over a very small geographic area, which may hold true for the whole $L$. praedator distribution range, while available morphological data insufficiently explain this diversity. Thus, we suggest the existence of several distinctive lineages or cryptic speciation in an 'L. praedator-complex', and a phylogenetic and taxonomic revision seems timely.

Tropical forests may harbor over 20 sympatric army ant species (Rettenmeyer et al. 1983; Longino et al. 2002). It is this very diversity which facilitates their enormous impact at the ecosystem level (Kaspari and O'Donnell 2003; Kaspari et al. 2011; O'Donnell et al. 2007), and we show that this diversity, particularly of the swarm-raiders, may be still underestimated. Our study, therefore, demonstrates the importance of detecting cryptic speciation as an essential element of understanding biodiversity and community dynamics in these ants and in general.

\section{Acknowledgments}

We thank D. Sanchez and O. Argüello from the ECOSUR research centre (El Colegio de la Frontera Sur, Tapachula, Mexico) for providing sampling permits and their help during field work, E. Stolle for assisting with morphological imaging, and D. Kleber and P. Leibe for 
technical support. Finances were granted by the Graduate Scholarship of Saxony Anhalt (MBB) and the Mexican-European FONCICYT 94293 Grant "MUTUAL" for travel expenses.

\section{References}

Avise JC (2000) Phylogeography: the history and formation of species. Harvard University Press, Cambridge, p 447

Barth MB, Moritz RFA, Pirk CWW, Kraus FB (2013) Male-biased dispersal promotes large scale gene flow in a subterranean army ant, Dorylus (Typhlopone) fulvus. Popul Ecol 55:523-533

Barth MB, Moritz RFA, Kraus FB (2014) The evolution of extreme polyandry in social insects: insights from army ants. PLoS One 9:e105621

Bensasson D, Zhang D, Hartl D, Hewitt G (2001) Mitochondrial pseudogenes: evolution's misplaced witnesses. Trends Ecol Evol 16:314-321

Berghoff SM, Kronauer DJC, Edwards KJ, Franks NR (2008) Dispersal and population structure of a New World predator, the army ant Eciton burchellii. J Evol Biol 21:1125-1132

Boomsma JJ, Ratnieks FLW (1996) Paternity in Eusocial Hymenoptera. Philos Trans R Soc Lond B Biol Sci 351:947-975

Borgmeier T (1955) Die Wanderameisen der Neotropischen Region. Stud Entomol 3:1-720

Brady SG (2003) Evolution of the army ant syndrome: the origin and long-term evolutionary stasis of a complex of behavioral and reproductive adaptations. Proc Natl Acad Sci USA 100:6575-6579

Denny AJ, Franks NR, Edwards KJ (2004) Eight highly polymorphic microsatellite markers for the army ant Eciton burchellii. Mol Ecol Notes 4:234-236

Fisher BL, Smith MA (2008) A revision of Malagasy species of Anochetus Mayr and Odontomachus Latreille (Hymenoptera: Formicidae). PLoS One 3:e1787

Fournier D, Tindo M, Kenne M, Mbenoun Masse PS, Van Bossche V, De Coninck E, Aron S (2012) Genetic structure, nestmate recognition and behaviour of two cryptic species of the invasive bigheaded ant Pheidole megacephala. PLoS One 7:e31480

Fowler HG (1979) Notes on Labidus praedator (Fr. Smith) in Paraguay (Hymenoptera: Formicidae: Dorylinae: Ecitonini). J Nat Hist 13:3-10

Franks NR (1982) Social insects in the aftermath of swarm raids of the army ant Eciton burchelli. In: Breed MD, Michener CD, Evans HE (eds) The biology of social insects: proceedings, ninth congress, International Union For The Study Of Social Insects, Boulder, Colorado, August 1982. Westview Press, Boulder, pp 275-279

Franks NR, Bossert WH (1983) The influence of swarm raiding army ants on the patchiness and diversity of a tropical leaf litter ant community. In: Sutton S, Whitmore T, Chadwick A (eds) Tropical rain forest: ecology and management. Blackwell Publishing, Oxford, pp 151-163

Gotwald WH (1995) Army ants: the biology of social predation. Comstock, Ithaca, p 302 
Goudet J (1995) FSTAT (version 1.2): a computer program to calculate F-statistics. J Hered 86:485486

Hall BG (2013) Building phylogenetic trees from molecular data with MEGA. Mol Biol Evol 30:12291235

Hebert PDN, Cywinska A, Ball SL, DeWaard JR (2003a) Biological identifications through DNA barcodes. Proc R Soc Lond B Biol Sci 270:313-321

Hebert PDN, Ratnasingham S, DeWaard JR (2003b) Barcoding animal life: cytochrome c oxidase subunit 1 divergences among closely related species. Proc R Soc Lond B Biol Sci 270:S96-S99

Hebert PDN, Penton EH, Burns JM, Janzen DH, Hallwachs W (2004) Ten species in one: DNA barcoding reveals cryptic species in the Neotropical skipper butterfly Astraptes fulgerator. Proc Natl Acad Sci USA 101:14812-14817

Jaffé R, Moritz RFA, Kraus FB (2009) Gene flow is maintained by polyandry and male dispersal in the army ant Eciton burchellii. Popul Ecol 51:227-236

Kalinowski ST (2005) HP-RARE 1.0: a computer program for performing rarefaction on measures of allelic richness. Mol Ecol Notes 5:187-189

Kaspari M, O'Donnell S (2003) High rates of army ant raids in the Neotropics and implications for ant colony and community structure. Evol Ecol Res 5:933-939

Kaspari M, Powell S, Lattke J, O'Donnell S (2011) Predation and patchiness in the tropical litter: do swarm-raiding army ants skim the cream or drain the bottle? J Anim Ecol 80:818-823

Kronauer DJC (2009) Recent advances in army ant biology (Hymenoptera: Formicidae). Myrmecol News 12:51-65

Kronauer DJC, Boomsma JJ, Gadau J (2004) Microsatellite markers for the driver ant Dorylus (Anomma) molestus. Mol Ecol Notes 4:289-290

Kronauer DJC, Johnson RA, Boomsma JJ (2007) The evolution of multiple mating in army ants. Evolution 61:413-422

Longino JT (2005) Ants of Costa Rica.

http://academic.evergreen.edu/projects/ants/AntsofCostaRica.html

Longino JT, Coddington J, Colwell RK (2002) The ant fauna of a tropical rain forest: estimating species richness three different ways. Ecology 83:689-702

Moilanen A, Sundström L, Pedersen JS (2004) MATESOFT: a program for deducing parental genotypes and estimating mating system statistics in haplodiploid species. Mol Ecol Notes 4:795797

Nei M, Kumar S (2000) Molecular evolution and phylogenetics. Oxford University Press, New York

O'Donnell S, Lattke J, Powell S, Kaspari M (2007) Army ants in four forests: geographic variation in raid rates and species composition. J Anim Ecol 76:580-589 
Rabeling C, Schultz TR, Pierce NE, Bacci M (2014) A social parasite evolved reproductive isolation from its fungus-growing ant host in sympatry. Curr Biol 24:2047-2052

Rettenmeyer CW (1963) Behavioral studies of army ants. Univ Kans Sci Bull 44:281-465

Rettenmeyer CW, Chadab-Crepet R, Naumann MG, Morales L (1983) Comparative foraging by neotropical army ants. In: Jaisson P (ed) Social insects in the tropics. Université Paris-Nord, Paris, pp 59-73

Schneirla TC (1971) Army ants: a study in social organization. W.H. Freeman, San Francisco, p 369

Seifert B (2009) Cryptic species in ants (Hymenoptera: Formicidae) revisited: we need a change in the alpha-taxonomic approach. Myrmecol News 12:149-166

Smith MA, Fisher BL, Hebert PDN (2005) DNA barcoding for effective biodiversity assessment of a hyperdiverse arthropod group: the ants of Madagascar. Philos Trans R Soc Lond B Biol Sci 360:18251834

Smith MA, Hallwachs W, Janzen DH (2014) Diversity and phylogenetic community structure of ants along a Costa Rican elevational gradient. Ecography 37:720-731

Tamura K, Nei M, Kumar S (2004) Prospects for inferring very large phylogenies by using the neighbor-joining method. Proc Natl Acad Sci USA 101:11030-11035

Tamura K, Peterson D, Peterson N, Stecher G, Nei M, Kumar S (2011) MEGA5: molecular evolutionary genetics analysis using maximum likelihood, evolutionary distance, and maximum parsimony methods. Mol Biol Evol 28:2731-2739

Walsh PS, Metzger DA, Higuchi R (1991) Chelex-100 as a medium for simple extraction of DNA for PCR-based typing from forensic material. Biotechniques 10:506-513

Watkins JF (1976) The identification and distribution of new world army ants (Dorylinae: Formicidae). Baylor University Press, Waco, p 102

Watkins JF (1982) Key to the army ants of Mexico. J Kans Entomol Soc 55:197-247 